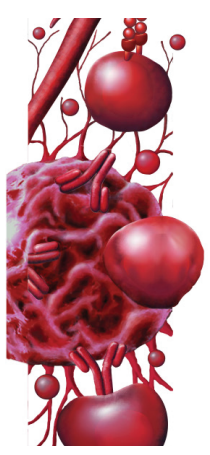

\title{
CAR T-cell therapy: opportunities and challenges
}

\begin{abstract}
A letter in response to: Milena Kalaitsidou, Gray Kueberuwa, Antje Schütt \& David Edward Gilham. CAR T-cell therapy: toxicity and the relevance of preclinical models. Immunotherapy 7(5), 487-497 (2015).
\end{abstract}

First draft submitted: 22 December 2015; Accepted for publication: 22 December 2015; Published online: 10 February 2016

Keywords: CAR T cells $\bullet$ toxicity $\bullet$ tumor microenvironment

In the May 2015 issue of Immunotherapy, Kalaitsidou et al. describe adoptive chimeric antigen receptor (CAR) engineered T-cell therapy from another angle: the issue of toxicity and novel strategies to overcome these toxicities [1].

From first through to fourth generation, CAR T-cell technology has developed rapidly. A patient's own $\mathrm{T}$ cells can be modified with a CAR, which not only targets the antigen that is expressed on the surface of the tumor cells specifically, but plays an independent role to the major histocompatibility complex. The results of some clinical trials have reversed conventional cognition for tumor treatment, at least the therapeutics for acute lymphoblastic leukemia [2-4]. A number of clinical reports suggested that CD19-CARs were notable for remission induced in patients with refractory and heavily pretreated $\mathrm{B}$ cell malignancies, and thereby they begin to appear to be the most successful design [5].

With the promising results in acute lymphoblastic leukemia, the complete remission rates increased from 70 to $90 \%$ [2-4]. Various CARs were designed for lymphomas and solid tumors [6,7], which were followed by a variety of side effects such as hypotension, respiratory distress, transient neurotoxicity, renal insufficiency and serious life-threat- ening events. The subsequent clinical trial results indicated that once the transgene products return to the body, CAR modified $T$ cells can selectively bind to the targeted antigens expressed on the tumor as well as the normal tissue, which also express these antigens at a relatively lower level. This also contributes to the adverse events after infusion. Adverse events of CAR T cells infusion can be summarized as 'on-target on-tumor, on-target off-tumor (Figure 1) and off-target off-tumor' [1]. On-target on-tumor reactions, including cytokine release syndrome and tumor lysis syndrome. They can be controlled with symptomatic, supportive strategy, glucocorticoid and even the anti-IL-6 receptor antibody Actemra (Tocilizumab) according to the grading of adverse reactions [8]. On-target off-tumor and off-target off-tumor events did not seem as easy to deal with. Persistent eradication of the healthy B-cell population induced by CD19-CAR could be managed by intravenous immune globulin [9]. However, the counter measure for targeted toxicities caused by other designed CARs remain limited. Even more, we cannot predict what events will occur after receiving the new CARs that we designed as the perfect pattern. Once the unpredictable events occur, we may activate the inhibitory signal pathway of CAR T cell (-iCAR) [10],
Wenlong Zhang

Department of Hematology \& Oncology, China-Japan Union Hospital of Jilin University, Changchun 130033, China Tel.: +8613504311151

wenlongzhang2016@sina.com
Future :\%: Medicine part of 


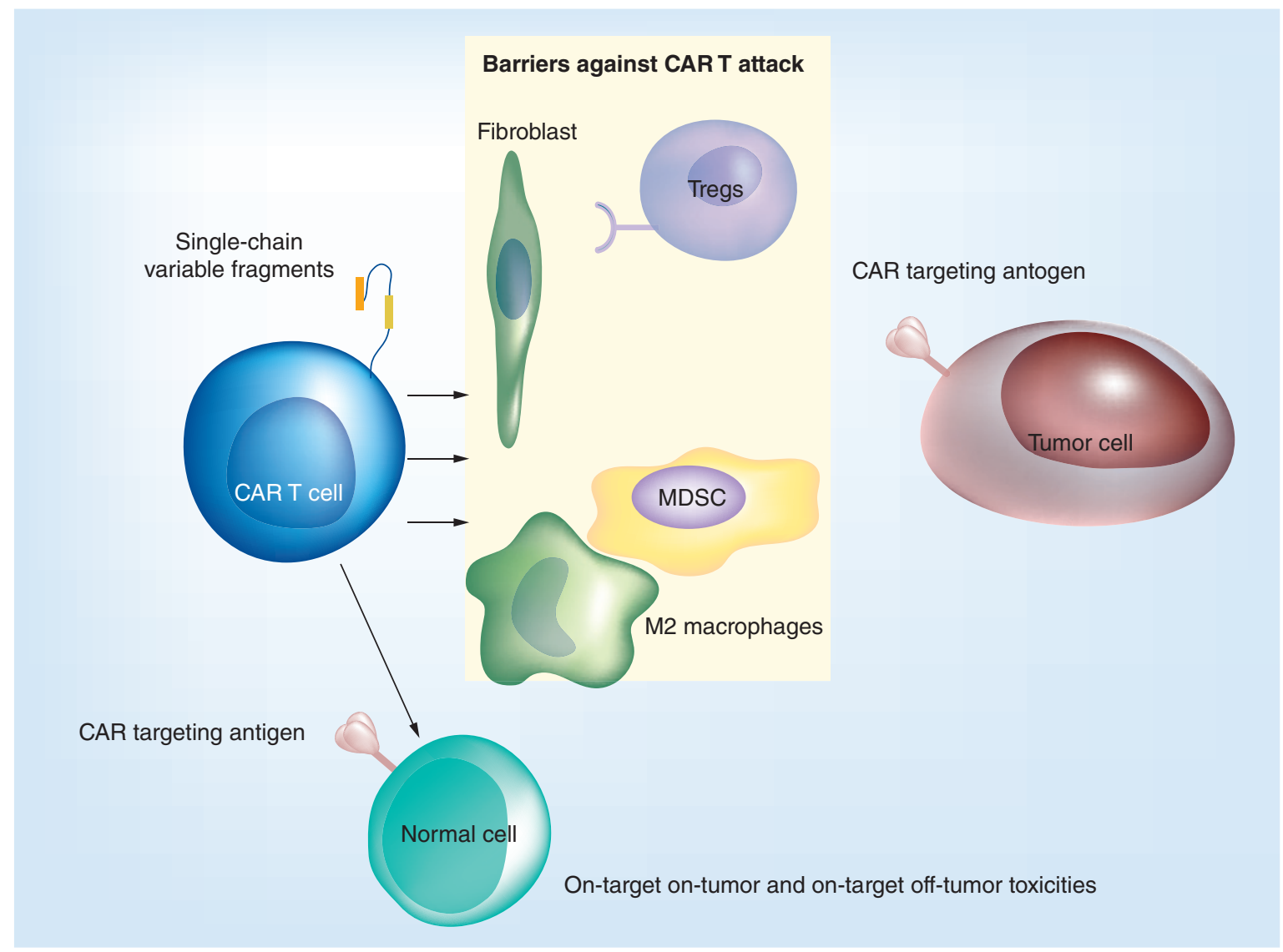

Figure 1. Toxicities and barriers of the CAR-T therapy. Single chain variable fragments also bind the antigen expressed by normal cells that may trigger the on-target on-tumor and on-target off-tumor toxicities. Tumor microenvironment presents itself as physical barriers and immune barriers to prevent $\mathrm{T}$ cells from directly contacting with targeted cells.

CAR: chimeric antigen receptor; Treg: Regulation T cell; MDSC: Myeloid-derived suppressor cell.

removing the toxic side effects at the expense of the therapeutic effect. Hence, if we do not get a handle on its toxicity, even this very potent therapy could selfdestruct. Novel tactics, not only for the encouraging results, but the controllable toxicities, remain to be one of the urgent but unmet needs today.

Aside from these toxicities, CAR T techniques were highly controversial and questioned whether they could bring a similar surprise in application of lymphoma and solid tumors. First, the tumor microenvironment, interdependent with lymphoma or solid tumors, presents as physical barriers to prevent $\mathrm{T}$ cells from direct contact with targeted cells [11] (Figure 1). Second, the peritumoral areas are surrounded by a tumor immune friendly environment which is composed by regulation T cells (Tregs), myeloid-derived suppressor cells (MDSCs) and M2 macrophage, all of which are working hard for weakening the function of $\mathrm{T}$ cells and promoting its exhaustion [12-14] (Figure 1). Third, the lack of homing receptors, CAR T cells are transformed into passing $\mathrm{T}$ cells rather than penetrating into the tumor [15]. Finally, repetitive CAR targeting may facilitate selective emergence of the CAR-negative oncocyte clone and promote immune escape. Emerging data indicate that CD19 proteins may be lost or truncated with therapeutic CD19-CAR T cells, therefore, some cases underwent a relapse or progression with the CD19 negative clone $[3,4]$. Those mentioned above constitute powerful challenges to immunotherapy targeting lymphoma and solid tumors.

CAR $\mathrm{T}$ technology, endowing the specific $\mathrm{T}$ cells ability to recognize and kill tumor cells directly, has brought the dawn of conquering malignant tumors, however, current issues to be solved are as follows. First, there is no uniform consensus on the dose of transfusion cells. Small dose infusions may not obtain the ideal curative effect, but induce tumor antigen deletion, then develop into immune escape; large dose infusions and serious tumor load will increase the cytokine release syndrome and tumor lysis syndrome. Whether we can seize the relationship between CAR T-cell infusion dosage and influence factors, including tumor load, effi- 
cacy and side effects, seems particularly critical. Second, the reasonable targets need to be set, which is possible to maximize the destruction of the tumor and minimize the toxicities. Third, the preconditioning scheme before infusion is needed. Chemotherapy induced lymphocyte depletion may reduce the anti-CAR immune response and induce bone marrow cytokine production that restores the immune cell populations and favors the activation of antitumor responses [15]. From another point of view, these cytokines can aggravate the cytokine release syndrome. Tregs and MDSCs are also increased after preprocessing, which is an unexpected by product result. Finally, due to the existence of tumor microenvironment, not only the physical barriers but the immune barriers for CAR-T therapy, combined with other targeting drugs or designed novel CARs have become a new trend [16-18].

\section{References}

1 Kalaitsidou M, Kueberuwa G, Schutt A, Gilham DE. CAR T-cell therapy: toxicity and the relevance of preclinical models. Immunotherapy7(5),487-497 (2015).

2 Davila ML, Riviere I, Wang X et al. Efficacy and toxicity management of 19-28z CAR T cell therapy in B cell acute lymphoblastic leukemia. Sci. Transl. Med. 6(224), $224 \mathrm{ra} 225$ (2014).

3 Maude SL, Frey N, Shaw PA et al. Chimeric antigen receptor $\mathrm{T}$ cells for sustained remissions in leukemia. N.Engl.J.M ed.371(16),1507-1517 (2014).

4 Lee DW, Kochenderfer JN, Stetler-Stevenson M et al. $\mathrm{T}$ cells expressing CD19 chimeric antigen receptors for acute lymphoblastic leukaemia in children and young adults: a Phase 1 dose-escalation trial. Lancet 385(9967), 517-528 (2015).

5 Maude SL, Teachey DT, Porter DL, Grupp SA. CD19targeted chimeric antigen receptor T-cell therapy for acute lymphoblastic leukemia. Blood 125(26), 4017-4023 (2015).

6 Prapa M, Caldrer S, Spano C et al. A novel anti-GD2/4-1BB chimeric antigen receptor triggers neuroblastoma cell killing. Oncotarget 6(28), 24884-24894 (2015).

7 Katz SC, Burga RA, Mccormack E et al. Phase I Hepatic immunotherapy for metastases study of intra-arterial chimeric antigen receptor-modified t-cell therapy for CEA+ liver metastases. Clin. Cancer Res. 21(14), 3149-3159 (2015).

8 Lee DW, Gardner R, Porter DL et al. Current concepts in the diagnosis and management of cytokine release syndrome. Blood 124(2), 188-195 (2014).

9 June CH, Maus MV, Plesa G et al. Engineered T cells for cancer therapy. Cancer Immunol. Immunother. 63(9), 969-975 (2014).
In conclusion, CAR T technology not only brings us spectacular opportunities, but also severe challenges. Precise targeting, accurate infusion dose, ideal efficacy and slight side effects will be considered as a starting point for the novel designed CARs, and a new era for tumor immunotherapy will emerge to overcome these hurdles mentioned above.

\section{Financial \& competing interests disclosure}

The author has no relevant affiliations or financial involvement with any organization or entity with a financial interest in or financial conflict with the subject matter or materials discussed in the manuscript. This includes employment, consultancies, honoraria, stock ownership or options, expert testimony, grants or patents received or pending, or royalties.

No writing assistance was utilized in the production of this manuscript.

10 Zhou X, Dotti G, Krance RA et al. Inducible caspase-9 suicide gene controls adverse effects from alloreplete $T$ cells after haploidentical stem cell transplantation. Blood 125(26), 4103-4113 (2015).

11 Papa S, Van Schalkwyk M, Maher J. Clinical evaluation of ErbB-targeted CAR T-cells, following intracavity delivery in patients with ErbB-expressing solid tumors. Methods Mol. Biol. 1317, 365-382 (2015).

12 Seliger B, Massa C. The dark side of dendritic cells: development and exploitation of tolerogenic activity that favor tumor outgrowth and immune escape. Front. Immunol. 4(419), 00419 (2013).

13 Tarhini AA, Zahoor H, Yearley JH et al. Tumor associated PD-L1 expression pattern in microscopically tumor positive sentinel lymph nodes in patients with melanoma. J. Transl. Med. 13(1), 319 (2015).

14 Lote H, Cafferkey C, Chau I. PD-1 and PD-L1 blockade in gastrointestinal malignancies. Cancer Treat. Rev. 21(15), 00168-00161 (2015).

15 Enblad G, Karlsson H, Loskog AS. CAR T-cell therapy: the role of physical barriers and immunosuppression in lymphoma. Hum. Gene Ther. 26(8), 498-505 (2015).

16 Chmielewski M, Hombach AA, Abken H. Of CARs and TRUCKs: chimeric antigen receptor (CAR) T cells engineered with an inducible cytokine to modulate the tumor stroma. Immunol. Rev. 257(1), 83-90 (2014).

17 Pegram HJ, Purdon TJ, Van Leeuwen DG et al. IL-12secreting CD19-targeted cord blood-derived T cells for the immunotherapy of B-cell acute lymphoblastic leukemia. Leukemia 29(2), 415-422 (2015).

18 Ankri C, Cohen CJ. Out of the bitter came forth sweet: activating CD28-dependent co-stimulation via PD-1 ligands. Oncoimmunology 3(1), e27399 (2014). 